\title{
Stiff-Person Syndrome Outpatient Rehabilitation: Case Report
}

\author{
Fayaz Khan ${ }^{1, \odot ~ M o h a m e d ~ F a i s a l ~ C h e v i d i k u n n a n ~}{ }^{1}$ Raghad Ahmad Almalki ${ }^{1}$ \\ Mawada Khaled Mirdad ${ }^{1} \quad$ Khadeeja Abdulaziz Nimatallah ${ }^{1}$ Shahad Al-Zahrani ${ }^{1}$ \\ Aysha Abdulmalek Alshareef ${ }^{2}$
}

\author{
${ }^{1}$ Department of Physical Therapy, Faculty of Medical Rehabilitation \\ Sciences, King Abdulaziz University, Jeddah, Saudi Arabia \\ ${ }^{2}$ Department of Neurology, King Abdulaziz University, Jeddah, \\ Saudi Arabia
}

\begin{abstract}
Address for correspondence Fayaz Khan, PT, PhD, Department of Physical Therapy, Faculty of Medical Rehabilitation Sciences,

King Abdulaziz University, Jeddah, Saudi Arabia

(e-mail: fayazrkhan@gmail.com,rkhan2@kau.edu.sa).
\end{abstract}

Abstract
Keywords
- stiff-person syndrome
- SPS
- rehabilitation
- physical therapy

Stiff-person syndrome (SPS) is a rare neurological disorder that causes muscle rigidity and stiffness of the trunk and proximal limb muscles, leading to movement difficulties and impaired function. Due to the rarity of the disease, studies on the benefit of rehabilitation for this disorder are quite limited. A 46-year-old female patient diagnosed with SPS complained of imbalance and movement difficulty. We prescribed therapeutic exercises aimed to reduce the stiffness of the trunk and proximal limbs and improve her function. Baseline measurement of the patient's range of motion, muscle power and tone, balance and functional abilities were taken pre- and post-program. Outcome measures showed a general improvement in the patient's muscle flexibility, balance, and functionality.

\section{Introduction}

Stiff-person syndrome "SPS" is a rare autoimmune neurological disorder that can affect one or two cases per million. ${ }^{1}$ Patients' symptoms usually start as pain and stiffness of the abdominal and lumbar region muscles and it can progress to the thorax and proximal upper and lower limbs muscles. ${ }^{2}$ The pathology's cause is still unclear. ${ }^{1}$

\section{Case Presentation}

\section{History}

Symptoms of the condition started around 2 years ago for our 46-year-old female patient with a progressing stiffness of the abdomen and proximal joints, worsening in the last 5 months and eventually she was unable to sit, stand, or walk independently. SPS was considered and tests were ordered and confirmed the diagnosis. The neurologist planned the patient for an immunotherapy and started her on clonazepam, baclofen, and steroids. She had no significant medical or surgical history.

\section{Physical Therapy Examination}

She was first seen by the therapist 1 month after she was diagnosed with SPS. Her chief complaints were regarding her balance and walking difficulty, as we noticed rigidity and slowness of her gait; she also complained about her shoulders' stiffness that interfaced with her job as a teacher. We aimed to assess the range of motion "ROM" of both her upper and lower extremities with a goniometer, muscle strength and tone, balance, activity of daily living (ADL), functional mobilities, and trunk functional assessment. On palpation, an obvious stiffness of the trunk and shoulder muscles was felt. The muscle flexibility of her upper extremities was severely reduced in her shoulders. Regarding her lower extremity, only her hip showed less than normal flexibility specially for flexion (-Table 1). We used modified Ashworth Scale for muscle tone ${ }^{3}$ and manual muscle testing for muscle power. ${ }^{4}$ Berg Balance Scale for balance assessment ${ }^{5}$ and ADL was assessed using the Barthel Index. ${ }^{6}$ Functional assessment was done with the Rivermead Mobility Index, ${ }^{7}$ and Trunk Impairment Scale for trunk assessment ${ }^{8}$ (-Table 2). 
Table 1 Range of motion measurements

\begin{tabular}{|c|c|c|c|c|}
\hline \multirow[t]{2}{*}{ AROM } & \multicolumn{2}{|c|}{ Right } & \multicolumn{2}{|c|}{ Left } \\
\hline & Before & After & Before & After \\
\hline Shoulder flexion & $0-30$ & $0-40$ & $(0-60)$ & $(0-70)$ \\
\hline Shoulder abduction & $0-55$ & $0-65$ & $(0-55)$ & $(0-55)$ \\
\hline Internal rotation & \multicolumn{2}{|c|}{ Normal } & \multicolumn{2}{|c|}{ Normal } \\
\hline External rotation & 0 & $0-30$ & 0 & $0-15$ \\
\hline Elbow flexion & $0-140$ & Full & $0-100$ & $0-110$ \\
\hline Wrist "all motions" & \multicolumn{2}{|c|}{ Normal } & \multicolumn{2}{|c|}{ Normal } \\
\hline Hip flexion & $0-40$ & $0-70$ & $0-65$ & $0-65$ \\
\hline Hip abduction & $0-20$ & $0-30$ & $0-30$ & $0-30$ \\
\hline Knee flexion & $0-115$ & Full & $0-125$ & Full \\
\hline Knee extension & \multicolumn{2}{|c|}{ Normal } & \multicolumn{2}{|c|}{ Normal } \\
\hline Ankle "all motions" & \multicolumn{2}{|c|}{ Normal } & \multicolumn{2}{|c|}{ Normal } \\
\hline
\end{tabular}

Abbreviation: AROM, active range of motion.

Table 2 Outcome measures at admission and discharge

\begin{tabular}{|l|l|l|}
\hline Outcome measure & Before & After \\
\hline Trunk Impairment Scale & $9 / 23$ & $18 / 23$ \\
\hline Rivermead Mobility Index & $6 / 15$ & $10 / 15$ \\
\hline Barthel Index & $75 / 100$ & $75 / 100$ \\
\hline Berg Balance Scale & $44 / 56$ & $48 / 56$ \\
\hline
\end{tabular}

\section{Intervention}

The patient had given consent before the intervention started and ethical approval was obtained from the faculty of applied medical sciences of the university. The main goal of physical therapy was to increase the patient's functionality and improve her balance by focusing on managing the muscle stiffness. The treatment plan was scheduled to be once a week with a home program. After 3 weeks of physical therapy, she underwent surgery after she was diagnosed with papillary thyroid carcinoma. So physical therapy was postponed for 1 week before we continued with our sessions for another 3 weeks, after that the patient had a head injury that led us to stop the sessions for a month before we could resume our program again for another 3 weeks before discharging her. The patient's sudden medical emergencies had no major effects on her health and required no subsequent rehab for them. So, in total the patient had 3 months of intermittent physical therapy with a total of nine sessions.

The sessions were focused on the muscle flexibility, and they were performed at different positions. All exercises were repeated for four times for each side of limb and each stretch was held for 30 seconds. Our patient also received manual therapy as she was given a mobilization with movement exercise for her shoulders on each session. All the previous exercises were prescribed as a home program twice a day in addition to 30 minutes of training on the stationary bike for general endurance improvement. Each session lasted for $\sim 2$ hours ( - Table 3 ).
Table 3 Rehabilitation program description

\begin{tabular}{|l|l|l|}
\hline $\begin{array}{l}\text { Starting } \\
\text { position }\end{array}$ & Exercise & Exercise description \\
\hline Supine & $\begin{array}{l}\text { Single knee } \\
\text { to chest }\end{array}$ & $\begin{array}{l}\text { One leg is bend and pressed } \\
\text { against the abdomen with the } \\
\text { hands }\end{array}$ \\
\cline { 2 - 3 } & $\begin{array}{l}\text { Lower trunk } \\
\text { rotation }\end{array}$ & $\begin{array}{l}\text { Both legs are bend, then they } \\
\text { bring them both to one side while } \\
\text { keeping the feet on bed }\end{array}$ \\
\cline { 2 - 3 } & Bridging & $\begin{array}{l}\text { While keeping both the knees } \\
\text { bend, raise the hips off the bed }\end{array}$ \\
\hline \multirow{2}{*}{ Sitting } & $\begin{array}{l}\text { Trunk } \\
\text { stretches }\end{array}$ & $\begin{array}{l}\text { Flexion, extension, side bending, } \\
\text { and rotation of the trunk }\end{array}$ \\
\cline { 2 - 3 } & $\begin{array}{l}\text { Balance } \\
\text { training }\end{array}$ & $\begin{array}{l}\text { Same tasks described in the Trunk } \\
\text { Impairment Scale }\end{array}$ \\
\hline Standing & $\begin{array}{l}\text { Single knee } \\
\text { to chest }\end{array}$ & $\begin{array}{l}\text { With hand support on stable sur- } \\
\text { face, bend one leg off the floor }\end{array}$ \\
\hline
\end{tabular}

\section{Measures and Outcomes}

After 3 months of physical therapy, the patient was reassessed, and the outcomes showed improvement in her muscle flexibility (-Table 1), while no change was seen in the muscle's power or the Barthel ADL assessment. Good improvement was reported in her balance and functional mobility and a great improvement was seen in trunk functionality as reported by the Trunk Impairment Scale. Generally, the patient's main improvements were seen in all her outcome measures except for her muscle strength and ADL assessment (-Table 2).

\section{Implications}

The patient's final outcomes showed an improvement in her functionality and balance caused by the improvement in her flexibility. A case report for an acute inpatient case showed improvement in the patient's functional outcomes. Their program also included stretching and strengthening exercises. ${ }^{9}$ A significant improvement in gait and lower back movement 
was reported in another case by applying ROM, stretching, and strengthening exercises. ${ }^{10}$ One detailed case report described a comprehensive and specific physical therapy program for their SPS case that improved her ROM, gait, and a reduction in pain and spasm. Her program mainly focused on her lower limbs flexibility and it consisted of a home program of strengthening, stretching, and ROM exercises for her lower limb, stationary bike, and soft tissue mobilization. ${ }^{11}$ Another report described the rehabilitation program at the inpatient rehabilitation hospital with daily sessions resulted in improvement in all the patient's functional outcomes. Self-stretching exercise were prescribed like the ones used in our case. They used knee to chest, sitting trunk flexion, lower trunk rotation, stretch of the hip's adductors and external rotators, and gastrocnemius stretching. ${ }^{12}$ And finally one report stated that stretching, ROM exercises for the stiff areas, and lower back exercises including knee to chest, pelvic tilt, and isometric abdominal exercises are beneficial for low back pain, hyperlordosis, mobility, gait and for improving the patient's function and their ability to perform their ADL. ${ }^{13}$

An important aspect of the outcomes is the fact that the patient was also on antispastic drugs and immunotherapy that might be attributed to this result, which is the case for all the SPS reports existed today making the exact effect of the physical therapy intervention to be hard to determine, but as SPS is thought to cause disability and functional limitation, rehabilitation via physical therapy to regain normal function is strongly advised. In conclusion, physical therapy rehabilitation appears to be beneficial for improving SPS patient's overall function.

\section{Institutional Review Board}

Faculty of Applied Medical Science, King Abdulaziz University, Jeddah, Saudi Arabia.

\section{Conflict of Interest}

None declared.

\section{References}

1 Hadavi S, Noyce AJ, Leslie RD, Giovannoni G. Stiff person syndrome. Pract Neurol 2011;11(5):272-282

2 Bhatti $A B$, Gazali ZA. Recent advances and review on treatment of stiff person syndrome in adults and pediatric patients. Cureus 2015;7(12):e427-e427

3 Charalambous CP, Interrater reliability of a modified Ashworth scale of muscle spasticity. Classic Papers in Orthopaedics. London: Springer; 2014 415-417

4 Wadsworth CT, Krishnan R, Sear M, Harrold J, Nielsen DH. Intrarater reliability of manual muscle testing and hand-held dynametric muscle testing. Phys Ther 1987;67(9):1342-1347

5 Godi M, Franchignoni F, Caligari M, Giordano A, Turcato AM, Nardone A. Comparison of reliability, validity, and responsiveness of the mini-BESTest and Berg Balance Scale in patients with balance disorders. Phys Ther 2013;93(2): 158-167

6 Collin C, Wade DT, Davies S, Horne V. The Barthel ADL Index: a reliability study. Int Disabil Stud 1988;10(2):61-63

7 Collen FM, Wade DT, Robb GF, Bradshaw CM. The Rivermead mobility index: a further development of the Rivermead motor assessment. Int Disabil Stud 1991;13(2):50-54

8 Verheyden G, Nieuwboer A, Mertin J. Preger R, Kiekens C, De Weerdt W. The Trunk Impairment Scale: a new tool to measure motor impairment of the trunk after stroke. Clin Rehabil 2004;18(3):326-334

9 Smith SR, Fu JB. Paraneoplastic stiff person syndrome: inpatient rehabilitation outcomes of a rare disease from two cancer rehabilitation programmes.J Rehabil Med 2016;48(7):639-642

10 Karaoglan B, Ote S, Koseoglu E, Ebru B, Balevi E. A stiff person case admitted physical medicine and rehabilitation outpatient clinic with low back pain. Turk J Phys Med Rehabil 2015;61(3):272

11 Hegyi CA. Physical therapist management of stiff person syndrome in a 24-year-old woman. Phys Ther 2011;91(9): 1403-1411

12 Potter K. Physical therapy during in-patient rehabilitation for a patient with stiff-person Syndrome. J Neurol Phys Ther 2006;30(1):28-38

13 Lorish TR, Thorsteinsson G, Howard FM Jr. Stiff-man syndrome updated. Mayo Clin Proc 1989;64(6):629-636 